\title{
Isolation and Structural Elucidation of Rosmarinic Acid by Nuclear Magnetic Resonance Spectroscopy
}

\author{
Elias Akoury
}

\author{
Faculty of Chemistry and Pharmacy \\ Ludwig-Maximilians-University Munich, Munich, Germany \\ eliasakoury@icloud.com
}

\begin{abstract}
Lemon balm is a herbal tea known for its therapeutic practice in soothing stomach cramps, indigestion and nausea. One of its chemical components is rosmarinic acid, known for its anti-inflammatory and antiviral activities in treating Herpes simplex. Despite the efforts, recovery and purification of rosmarinic acid from Lemon balm in high yields has not been entirely successful. Here, we report the aqueous extraction of rosmarinic acid from dried leaves of Lemon balm using Soxhlet distillation after optimizing the conditions with various organic solvents. We have isolated rosmarinic acid under optimum process conditions and high yield (96\%) and elucidated its structure by NMR spectroscopy.
\end{abstract}

Keywords:Rosmarinic acid, Lemon Balm, Natural Product Isolation, NMR spectroscopy

\section{INTRODUCTION}

Melissa officinalis Labiatae is an edible herb native to the Mediterranean and Eastern Europe. It is one of the oldest and still most popular medicinal plants. Lemon Balm is the common name of Melissa officinalis. This plant naturalizes easily, reproduces by underground stems and grows up to $45 \mathrm{~cm}$ in height. The herbal tea can be used therapeutically for soothing stomach cramps, indigestion and nausea. It is also lightly stimulating and a good tea to drink when studying. Melissa should be distilled before it flowers. The entire above ground plant is taken, the heavier stems discarded and steam-distilled to obtain the hydrosols. The major components of lemon balm are caffeic acid, the flavonoids: luteolin-7-0-glucoside, isoquercitrin, apigenin-7-0-glucoside, ahe essential oil $(0.19 \%)$ from the flowers contains various aldehydes including citronellal (22-36\%), citral (11-26\%), $\beta$-caryophyllene (10-15\%), and germacrene D (10-20\%).(Mulkens \& Kapetanidis, 1987) The essential oil is hypotensive, a calming sedative and anti-inflammatory. It is used for insomnia, hysteria and irritability by inhalation and will relieve a cold sore if applied externally.(Wu, Hong, Klauck, Lin, \& Efferth, 2015)

Rosmarinic acid has been known as "Labiatengerbstof" even before its chemical structure was elucidated. It is a tannin-like compound, described as a depside of caffeic acid. Originally identified in rosemary (Rosmarinus officinalis $L$.), the structure was elucidated as an ester of caffeic acid and 3-(3,4-dihydroxyphenyl)lactic acid. (Sanbongi et al., 2003; Youn et al., 2003) The compound has been reported to occur in several taxonomically non-related families of the plant kingdom. It has attracted much attention since it was identified to be the main compound responsible for the antiviral activity of lemon balm in treating Herpes simplex.(Sanchez-Medina et al., 2007) Rosmarinic acid is defined as a phenolic acid or an ester of caffeic acid. It has antioxidant, anti-inflammatory and antimicrobial activities.(Zhou et al., 2016) Its antioxidant activity is stronger than that of vitamin $\mathrm{E}$ and helps to prevent cell damage caused by free radicals; thereby reducing the risk for cancer and arthrosclerosis. It is also used for its anti-allergic activity, and unlike antihistamines, rosmarinic acid prevents the activation of immune responder cells, which cause swelling and fluid formation. Moreover, rosmarinic acid is used to treat peptic ulcers, arthritis, cataract, cancer, rheumatoid arthritis and bronchial asthma.(Ferreira Jde et al., 2015; Kantar Gok et al., 2015; Mushtaq et al., 2015) Also, it sustains normal basophile, cytokine and eosinophile 
functions. Despite the efforts, the recovery and purification of rosmarinic acid from Lemon balm in high yields has not been entirely successful. Here, we report the aqueous extraction of rosmarinic acid from dried leaves of Lemon balm using Soxhlet distillation under optimum process conditions and high yield (96\%) to elucidated its structure by NMR spectroscopy.

\section{Materials AND METHOD}

\section{Isolation of Rosmarinic Acid}

Dried lemon balm leaves were finely grounded to a net weight of $140 \mathrm{~g}$ and introduced in a cellulose thimble for Soxhlet extraction. The sample was washed with $500 \mathrm{~mL}$-hexane for 10 hours to discard all fatty materials and reflux with 1:1 $\mathrm{H}_{2} \mathrm{O}$ /ethanol was performed twice for 10 hours. The resulting dark green solution contained rosmarinic acid and other natural compounds. The water-soluble compounds were discarded after n-butanol extraction and the dried material containing rosmarinic acid was then dissolved in 3:1 $\mathrm{H}_{2} \mathrm{O}$ /toluene solution to discard non-polar organic products. The $\mathrm{pH}$ of the water fraction was adjusted to 4.5 and fractionated twice with methyl tert-butyl ether (MTBE) to isolate rosmarinic acid from other organic compounds. The adjustment of $\mathrm{pH}$ was carefully controlled since rosmarinic acid is insoluble in water at $\mathrm{pH}$ 3.7. The dried material was then dissolved in $300 \mathrm{~mL}$ distilled water and partitioned twice with $100 \mathrm{~mL}$ toluene to isolate non-polar organic products from rosmarinic acid. The toluene upper level was discarded and the $\mathrm{pH}$ of the water fraction was increased from $\mathrm{pH} 3.87$ to 4.5 using $10^{-2} \mathrm{M} \mathrm{NaOH}$. At this $\mathrm{pH}$, rosmarinic acid remains in water and other products are discarded after two extractions with $100 \mathrm{~mL}$ MTBE. The aqueous fraction was then adjusted to pH 3.75 and partitioned with five fractions of $100 \mathrm{~mL}$ MTBE. At this $\mathrm{pH}$, rosmarinic acid becomes insoluble in water but soluble in MTBE. This latter fraction is collected and brought to dryness. The dried material was then dissolved in hot water, active charcoal added and the solution stirred for 15 minutes then filtrated and refrigerated. The aqueous extraction of rosmarinic acid using Soxhlet distillation under optimal conditions was of high yield (96\%).

\section{NMR Spectroscopy}

The NMR sample was prepared by dissolving rosmarinic acid in $500 \mu \mathrm{L}$ in deuterated methanol $\mathrm{CD}_{3} \mathrm{OD}$. Spectra were internally referenced to the singlet resonance of 4,4-dimethyl-4-silapentane-1-sulfonic acid (DSS) at 0 ppm. Spectra were recorded on a BRUKER AVANCE DRX $400 \mathrm{MHz}$ NMR spectrometer. All the spectra were recorded using a sample with a concentration of $1.6 \mathrm{mM}$ at $300 \mathrm{~K}$. The experiments used were COrrelation SpectroscopY (COSY), Nuclear Overhauser Effect SpectroscopY (NOESY), Heteronuclear Single Coherence Correlation (13C-HSQC), Heteronuclear Multiple Bond Correlation (13C-HMBC). 1D 1H NMR parameters included 2 second acquisition time, 1.5 second relaxation delay, 16 scans and a total time of 1 minute. Variations on the 1D experiment include 1D NOE, selective decoupling solvent saturation and T1 determination. 1D 13C NMR parameters included 0.8 second acquisition time with proton decoupling, 2.0 second relaxation delay with NOE enhancement, 192 scans and a total time of 10 minutes. All 2D NMR experiments parameters included 1.5 second relaxation delay, 1 to 8 scans and a total time between 5 minutes and 1 hour.

\section{RESULTS AND DisCUSSION}

Among the organic solvents tested for rosmarinic acid recovery from its aqueous plant extracts, methyl tert-butyl ether (MTBE) and methanol were found to be most suitable and no other solvent among the tested ones (toluene, acitonitrile, petrol ether, ethanol, water) showed fair solubility in attempt to recover rosmarinic acid crystals. However, $\mathrm{pH}$ controlled extraction showed a high success of purification. Column chromatography was tested using the mobile phase methanol on Sephadex LH 20 material for retaining rosmarinic acid. This showed good separation although minor hydrolysis had been observed. 
We then investigated the structural elucidation of rosmarinic acid by NMR spectroscopy. The structure of rosmarinic acid $\left(\mathrm{C}_{18} \mathrm{H}_{16} \mathrm{O}_{8}\right)$ incorporates two unsaturated 6-membered rings (C1-C6) and $\mathrm{C1}^{\prime}$ - $\left.\mathrm{C}^{\prime}\right)$, a double bond ( $\left.\mathrm{C}^{\prime}-\mathrm{C}^{\prime}\right)$ an ester group ( $\left.\mathrm{C}^{\prime}\right)$, a carboxyl group (C9) and four hydroxyl groups (C3, C4, C3', C4'). Figure 2 represents the ${ }^{1} \mathrm{H}$ spectrum of rosmarinic acid dissolved in $\mathrm{CD}_{3} \mathrm{OD}$. Residual peaks at $\delta 4.75 \mathrm{ppm}$ and $\delta$ $3.15 \mathrm{ppm}$ correspond to water and to $\mathrm{CH}_{3}$ of methanol, respectively. The structure comprises $9 \mathrm{CH}$ groups, $1 \mathrm{CH}_{2}$ and 8 quaternary carbons Cq; all were visible on the ${ }^{13} \mathrm{C}$ spectrum of rosmarinic acid (Figure 2). The peak at $\delta 48.99 \mathrm{ppm}$ corresponds to the deuterated methanol. Figure 3 shows the $2 \mathrm{D}{ }^{1} \mathrm{H}-{ }^{1} \mathrm{H}$ COSY correlation spectrum of rosmarinic acid. This experiment gives information about pairs of protons that are J-coupled and usually indicates that the protons are on adjacent carbons, 2 or 3 bonds away. $2 \mathrm{D}{ }^{1} \mathrm{H}-{ }^{1} \mathrm{H}$ NOESY correlation spectrum of rosmarinic acid showing proton-proton through-space interactions via NOE (Figure 3). It gives information about pairs of protons that are close in space, being less than $5 \mathrm{~A}^{\circ}$ apart. Moreover, the $2 \mathrm{D}^{1} \mathrm{H}^{-13} \mathrm{C}$ HSQC spectrum, shown in Figure 4, represents the direct proton-carbon J-couplings of the different protons and carbons present in rosmarinic acid. Furthermore, the 2D ${ }^{1} \mathrm{H}-{ }^{13} \mathrm{C}$ HMBC spectrum gives information about weak proton-carbon J-couplings, indicating that the proton is two, three, or four bonds away from the carbon. This reveals which protons are near to different carbons, without being directly bonded to. HMBC, in conjunction with HSQC, can give an enormous amount of information about molecular structure, since the long-range proton-carbon correlations can include quaternary carbons, in addition to protonated carbons. All chemical shifts are represented in Table 1 . The obtained data are in agreement with previously published data. (Exarchou, Troganis, Gerothanassis, Tsimidou, \& Boskou, 2001; Takeda, Tsuji, Matsumiya, \& Kubo, 2002) The ${ }^{1} \mathrm{H}$ NMR spectrum of rosmarinic acid showed two doublets at 7.39 and 6.11 which on the basis of the observed large proton-proton coupling $(U=15.9 \mathrm{~Hz}$ ) were assigned to a pair of trans- olefinic protons. In addition, there were two ABX-spin systems observed in the aromatic region, which were assignable to the two discrete sets of protons of the 3,4-dihydroxyphenyl unit. It is worth mentioning that the other peaks are attributed mainly to caffeic acid, which is either obtained as a by- product of rosmarinic acid hydrolysis or might naturally originates from the plant itself.

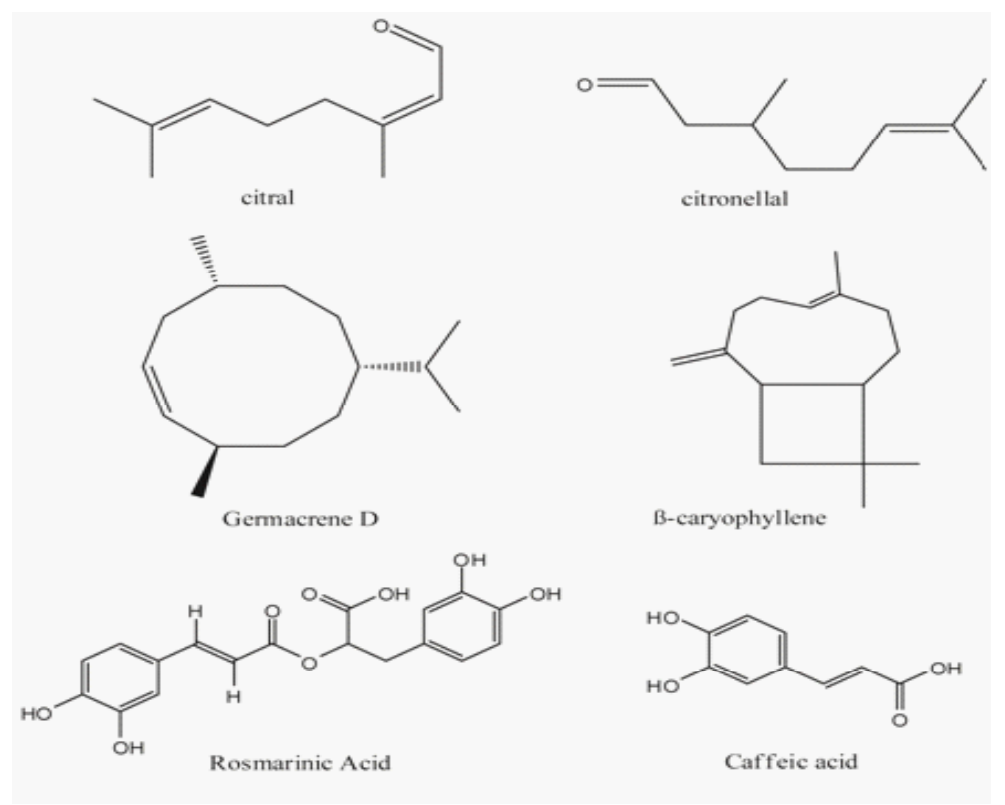

Fig 1. Chemical Structures of the major compounds found in Lemon Balm 
Isolation and Structural Elucidation of Rosmarinic Acid by Nuclear Magnetic Resonance Spectroscopy

A
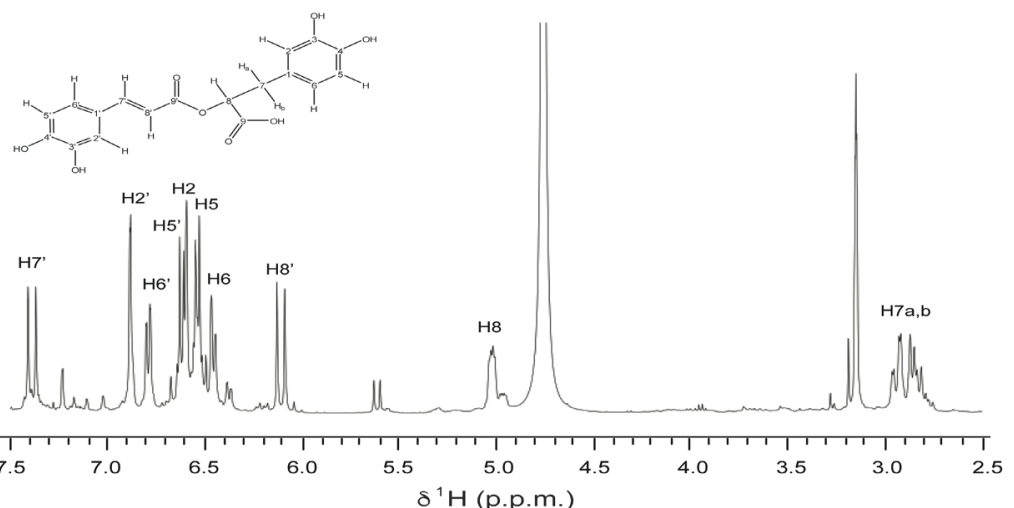

B
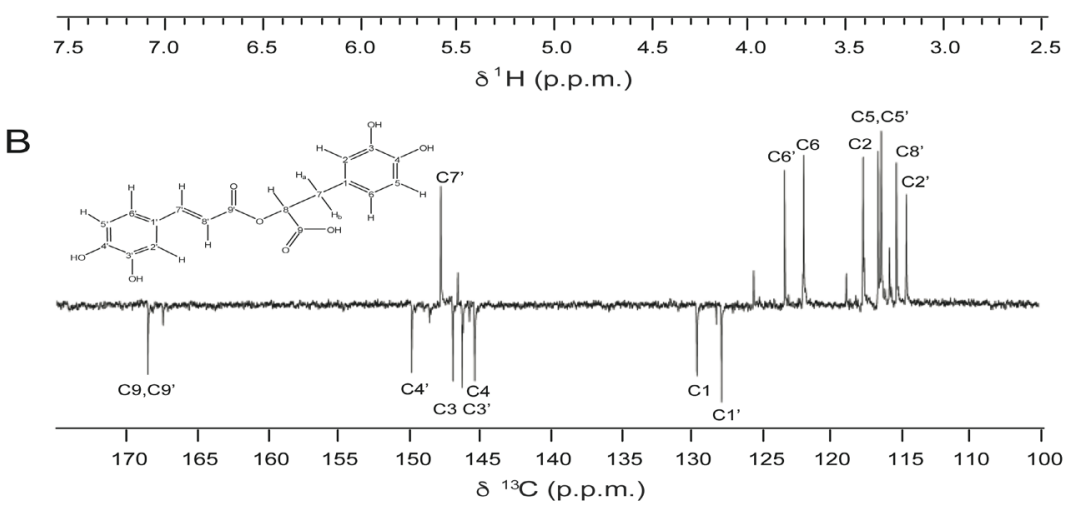

Fig 2. (A) 1D 1H NMR and (B) 1D 13C NMR spectra of Rosmarinic Acid
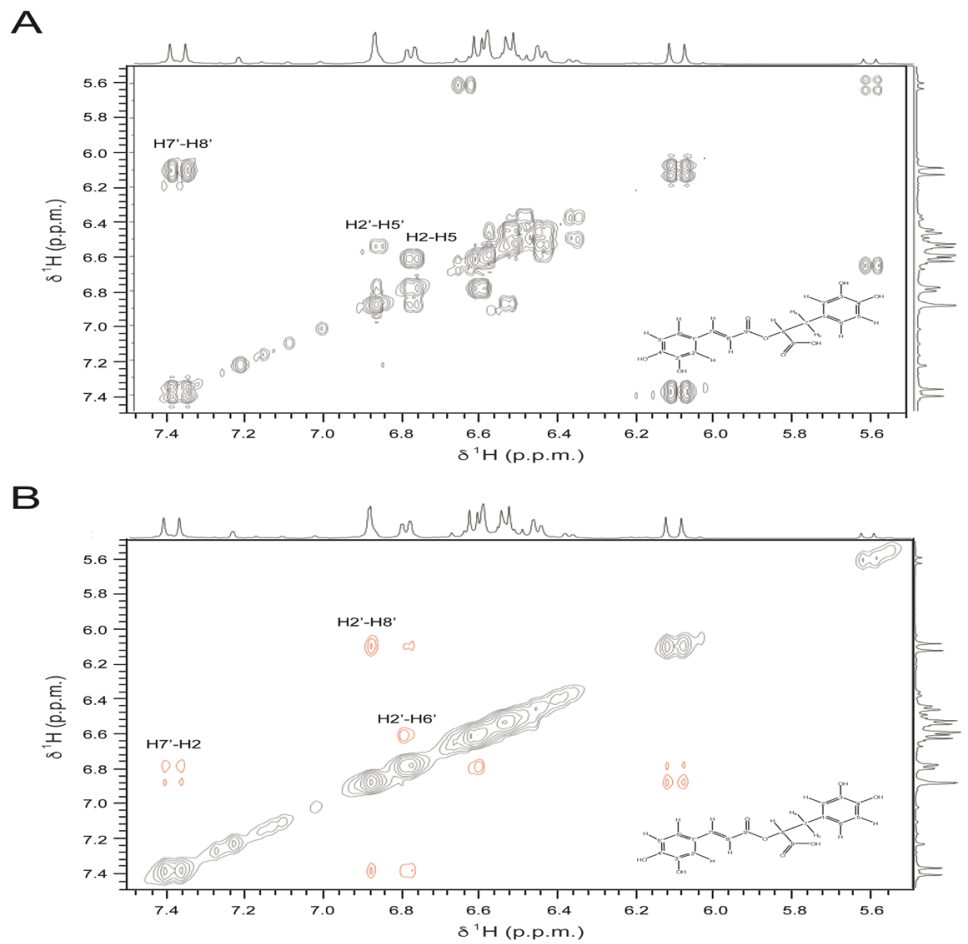

Fig 3. (A) 2D $1 H$-1H COSY and (B) 2D $1 H-1 H$ NOESY spectra of Rosmarinic Acid 
A

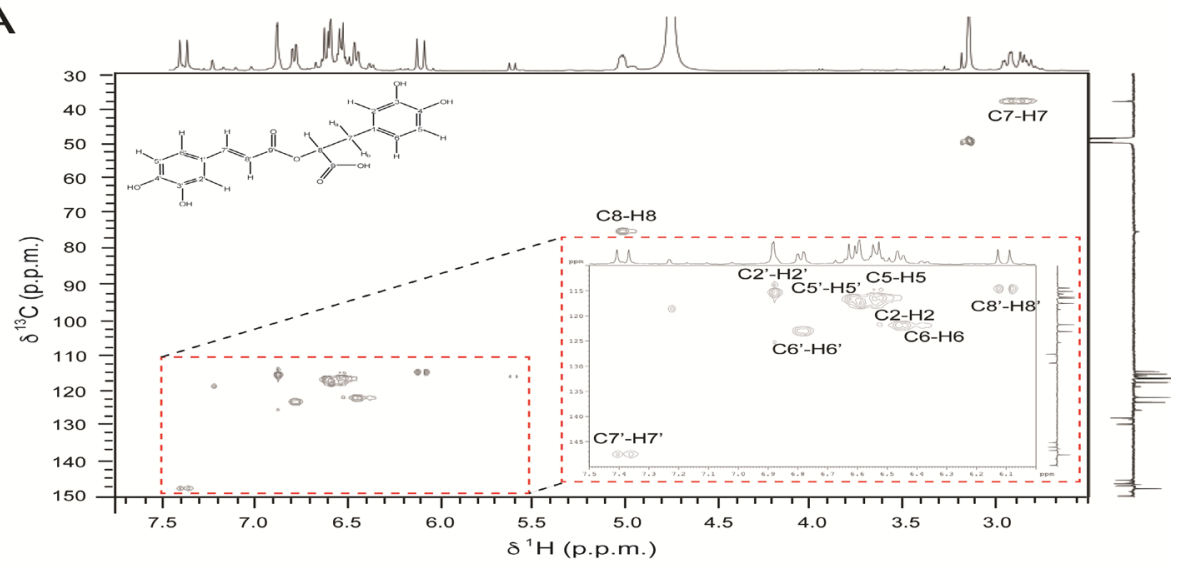

B

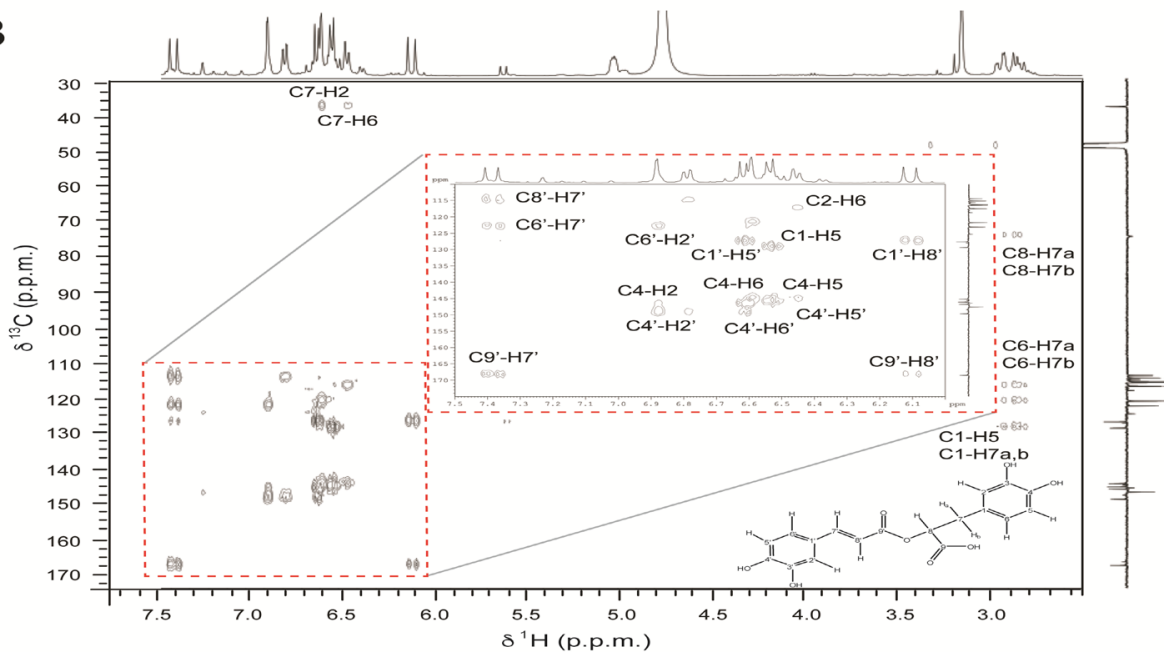

Fig 4. (A) 2D $1 H$-13C HSQC and (B) 2D 1 H -13C HMBC spectra of Rosmarinic Acid

Table 1. 1H NMR and 13C NMR Chemical Shifts of Rosmarinic Acid.

\begin{tabular}{|c|c|c|c|c|c|c|}
\hline Position & ${ }^{1} \mathbf{H}$ & ${ }^{13} \mathbf{C}$ & & Position & ${ }^{1} \mathbf{H}$ & ${ }^{13} \mathbf{C}$ \\
\hline 1 & - & 129.4 & & $1^{\prime}$ & - & 127.6 \\
\hline 2 & 6.59 & 117.5 & & $2^{\prime}$ & 6.88 & 114.5 \\
\hline 3 & - & 146.1 & & $3^{\prime}$ & - & 146.8 \\
\hline 4 & - & 145.2 & & $4^{\prime}$ & - & 149.7 \\
\hline 5 & 6.54 & 116.5 & & $5^{\prime}$ & 6.61 & 116.2 \\
\hline 6 & 6.47 & 121.8 & & $6^{\prime}$ & 6.79 & 123.1 \\
\hline $7 \mathrm{a}, \mathrm{b}$ & $2.85,2.93$ & 37.9 & & $7^{\prime}$ & 7.39 & 147.6 \\
\hline 8 & 5.01 & 74.8 & & $8^{\prime}$ & 6.11 & 115.2 \\
\hline 9 & - & 167.4 & & $9^{\prime}$ & - & 168.5 \\
\hline
\end{tabular}


We report the aqueous extraction of rosmarinic acid from dried leaves of Lemon balm and its purification in high yields with optimal solvents conditions. We have fully elucidated the structure of rosmarinic acid by NMR spectroscopy and shed lights on the conformational and dynamic stability of the molecule. Among the organic solvents tested for rosmarinic acid recovery from its aqueous plant extracts, methyl tert-butyl ether (MTBE) and methanol were found to be most suitable. Despite the successful purification, an appropriate mobile phase and an adaptable fixed chromatographic bed could be further investigated, both insuring no hydrolysis and high purity. In this study, we have reached successful attempts to grasp highest degrees of purification, to effectively discard by-products, and to reliably elucidate the chemical structure of one of the most important antioxidant, anti-inflammatory and antimicrobial compounds, rosmarinic acid.

\section{REFERENCES}

1. Exarchou, V., Troganis, A., Gerothanassis, I. P., Tsimidou, M., \& Boskou, D. (2001). Identification and quantification of caffeic and rosmarinic acid in complex plant extracts by the use of variable-temperature two-dimensional nuclear magnetic resonance spectroscopy. J Agric Food Chem, 49(1), 2-8.

2. Ferreira Jde, L., Chahud, F., Ramalho, L. N., Modulo, C. M., Vieira, L. C., Reinach, P. S., . . Paula, J. S. (2015). Rosmarinic Acid Suppresses Subconjunctival Neovascularization in Experimental Glaucoma Surgery. Curr Eye Res, 40(11), 1134-1140. doi:10.3109/02713683.2014.980911

3. Kantar Gok, D., Ozturk, N., Er, H., Aslan, M., Demir, N., Derin, N., .. Yargicoglu, P. (2015). Effects of rosmarinic acid on cognitive and biochemical alterations in ovariectomized rats treated with D-galactose. Folia Histochem Cytobiol, 53(4), 283-293. doi:10.5603/fhc.a2015.0034

4. Mulkens, A., \& Kapetanidis, I. (1987). [Flavonoids of the leaves of Melissa officinalis L. (Lamiaceae)]. Pharm Acta Helv, 62(1), 19-22.

5. Mushtaq, N., Schmatz, R., Ahmed, M., Pereira, L. B., da Costa, P., Reichert, K. P., . . Schetinger, M. R. (2015). Protective effect of rosmarinic acid against oxidative stress biomarkers in liver and kidney of strepotozotocin-induced diabetic rats. J Physiol Biochem, 71(4), 743-751. doi:10.1007/s13105-015-0438-4

6. Sanbongi, C., Takano, H., Osakabe, N., Sasa, N., Natsume, M., Yanagisawa, R., . . Yoshikawa, T. (2003). Rosmarinic acid inhibits lung injury induced by diesel exhaust particles. Free Radic Biol Med, 34(8), 1060-1069.

7. Sanchez-Medina, A., Etheridge, C. J., Hawkes, G. E., Hylands, P. J., Pendry, B. A., Hughes, M. J., \& Corcoran, 0. (2007). Comparison of rosmarinic acid content in commercial tinctures produced from fresh and dried lemon balm (Melissa officinalis). J Pharm Pharm Sci, 10(4), 455-463.

8. Takeda, H., Tsuji, M., Matsumiya, T., \& Kubo, M. (2002). Identification of rosmarinic acid as a novel antidepressive substance in the leaves of Perilla frutescens Britton var. acuta Kudo (Perillae Herba). Nihon Shinkei Seishin Yakurigaku Zasshi, 22(1), 15-22.

9. Wu, C. F., Hong, C., Klauck, S. M., Lin, Y. L., \& Efferth, T. (2015). Molecular mechanisms of rosmarinic acid from Salvia miltiorrhiza in acute lymphoblastic leukemia cells. J Ethnopharmacol, 176, 55-68. doi:10.1016/j. jep.2015.10.020 
Isolation and Structural Elucidation of Rosmarinic Acid by Nuclear Magnetic Resonance Spectroscopy

10. Youn, J., Lee, K. H., Won, J., Huh, S. J., Yun, H. S., Cho, W. G., \& Paik, D. J. (2003). Beneficial effects of rosmarinic acid on suppression of collagen induced arthritis. J Rheumatol, 30(6), 1203-1207.

11. Zhou, M. W., Jiang, R. H., Kim, K. D., Lee, J. H., Kim, C. D., Yin, W. T., \& Lee, J. H. (2016). Rosmarinic acid inhibits poly(I:C)-induced inflammatory reaction of epidermal keratinocytes. Life Sci, 155, 189-194. doi:10.1016/j. lfs.2016.05.023

Citation: Elias Akoury, "Isolation and Structural Elucidation of Rosmarinic Acid by Nuclear Magnetic Resonance Spectroscopy”. American Research Journal of Chemistry, 1(1); pp:17-23.

Copyright (C) Elias Akoury, This is an open access article distributed under the Creative Commons Attribution License, which permits unrestricted use, distribution, and reproduction in any medium, provided the original work is properly cited. 